Applications of Surface Science 5 (1980) 321-331

(c) North-Holland Publishing Company

\title{
THE INTERACTION OF OXYGEN WITH Ni(100) AND THE REDUCTION OF THE SURFACE OXIDE BY HYDROGEN
}

\author{
P.K. de BOKX, F. LABOHM, O.L.J. GIJZEMAN, G.A. BOOTSMA * and J.W. GEUS \\ Van 't Hoff Laboratory, University of Utrecht, Utrecht, The Netherlands
}

Received 27 November 1979

\begin{abstract}
The interaction of oxygen with $\mathrm{Ni}(100)$ has been studied with ellipsometry, Auger electron spectroscopy and low energy electron diffraction. The observations of other workers are completely confirmed. The same techniques and procedures have been used to study the reduction of the surface oxide with hydrogen. It is found that the reduction reaction requires hydrogen atoms, adsorbed on exposed nickel atoms. At temperatures between 150 and $250^{\circ} \mathrm{C}$ and hydrogen pressures up to $10^{-5}$ Torr, the reduction proceeds via a nucleation and growth type mechanism, with an activation energy of $23 \mathrm{kcal} / \mathrm{mol}$.
\end{abstract}

\section{Introduction}

The interaction of oxygen with nickel single crystals has been studied by many authors [1-8]. The low index faces behave quite similarly. First, a chemisorption stage is observed with Auger electron spectroscopy [1-3], with a saturation coverage of $\theta=0.5$ for $\mathrm{Ni}(100)$, followed by the growth of oxide islands of two to three layers of $\mathrm{NiO}$. The same observations have been made with photoelectron spectroscopy [4,6]. LEED and RHEED studies [3,5] have revealed the existence of $\mathrm{p} 2 \times 2$ and $\mathrm{c} 2 \times 2$ atomic overlayers in the chemisorption and a complicated "NiO" overlayer in the subsequent oxidation stage.

The reduction of the surface $\mathrm{NiO}$ with hydrogen seems to be less well studied, although an extensive literature on the reduction of bulk or supported $\mathrm{NiO}$ particles exists [9-12]. In these systems the reduction proceeds via a nucleation and growth mechanism described by the theory of Avrami [13], though kinetic studies can be hampered by transport problems [14].

In the present paper we report on our results on the oxidation of $\mathrm{Ni}(100)$ with $\mathrm{O}_{2}$ and the subsequent reduction of the surface nickel oxide with $\mathrm{H}_{2}$. Ellipsometry was chosen as the means to monitor the surface oxygen coverage, which allows continuous accumulation of kinetic data as in previous work $[15,16]$. Auger electron

\footnotetext{
* Permanent address: Department of Applied Physics, Twente University of Technology, 7500 AE Enschede, The Netherlands.
} 
spectroscopy and LEED have been used to correlate the observed ellipsometric signals with surface coverages. A more detailed interpretation of ellipsometric data in the (sub)monolayer region is difficult as will be shown in a future publication [17].

\section{Experimental}

The experiments were carried out in the vacuum system described previously [18], with facilities for AES-LEED and simultaneous ellipsometry measurements. The base pressure was $\approx 10^{-10}$ Torr. The crystal was the same as that used in previous experiments [19]. After sputter-cleaning and annealing the crystal a small amount of carbon always segregated to the surface $(\approx 0.05$ monolayer $)$.

Ellipsometry was done at an angle of incidence $\phi_{0}=68.2^{\circ}, \lambda=6328 \AA$ with either two-zone or off-null irradiance measurements. The values obtained for the clean $\mathrm{Ni}(100)$ crystal $(\bar{\Delta}=133.08 \pm 0.12, \bar{\psi}=34.53 \pm 0.04)$ lead to optical constants $n$ $i k=2.33 \pm 0.04-i(4.30 \pm 0.08)$ which are in good agreement with those reported previously for $\mathrm{Ni}(110)$ [18].

\section{Results}

The effect of adsorption of oxygen on $\mathrm{Ni}(100)$ on the change in the ellipsometric parameter $\Delta$ is shown in fig. 1 . It can be seen that the value of $\Delta$ is virtually constant

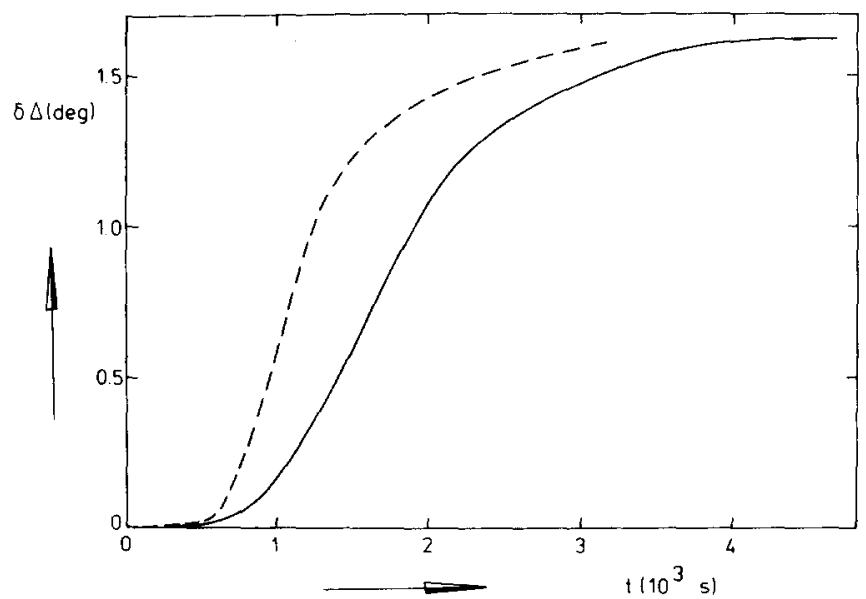

Fig. 1. Typical off-null curves for the reaction of oxygen with Ni(100). Note the absence of any change in $\Delta$ in the chemisorption stage $(\leqslant 40 \mathrm{~L})$. Full curve: $T=25^{\circ} \mathrm{C}, p=7.3 \times 10^{-8}$ Torr; dashed curve: $T=50^{\circ} \mathrm{C}, p=1.1 \times 10^{-7}$ Torr. 
up to an exposure of $40 \mathrm{~L}\left(1 \mathrm{~L}=10^{-6}\right.$ Torr $\left.\mathrm{s}=1.3310^{-4} \mathrm{~Pa} \mathrm{~s}\right)$, after which a sizeable change occurs up to $\delta \Delta=\bar{\Delta}-\Delta=1.65 \mathrm{deg}$. This change reflects the formation of a surface nickel oxide by incorporation of oxygen and reconstruction of the $\mathrm{Ni}$ surface, as evidenced by parallel AES-LEED measurements. Fig. 2 shows the normalised oxygen Auger peak heights $\left(h_{\mathrm{O}} / h_{\mathrm{Ni}}\right)$, taken at $515 \mathrm{eV}$ for oxygen and 848 $\mathrm{eV}$ for nickel, versus $\delta \Delta$ and $\delta \psi$ measured after adsorption of increasing amounts of oxygen by the crystal. Between 25 and $150^{\circ} \mathrm{C}$ the slope of the straight part of the curve is independent of the crystal temperature. The non-linear part of the calibration curve $\left(h_{\mathrm{O}} / h_{\mathrm{Ni}}<0.7\right)$ corresponds to the formation of the $\mathrm{Ni}(100) \mathrm{c}(2 \times 2) \mathrm{O}$ structure as deduced from LEED photographs. It may thus be concluded that in the reconstruction region $h_{\mathrm{O}} / h_{\mathrm{Ni}}=(0.94 \pm 0.05) \delta \Delta$, that is, $\delta \Delta$ is (roughly) proportional to the amount of incorporated oxygen. In the pressure range $10^{-8}$ to $10^{-6}$ Torr the observed changes in $\Delta$ depend only on the exposure.

The reaction of hydrogen with oxidised $\mathrm{Ni}(100)$ was studied at crystal tempera. tures between 150 and $250^{\circ} \mathrm{C}$ and hydrogen pressures of $10^{-6}$ to $10^{-4}$ Torr. The clean, annealed, crystal had previously been exposed to oxygen at temperatures below $100^{\circ} \mathrm{C}$. Interaction with hydrogen caused the oxygen coverage to decrease as shown in fig. 3 . In the absence of hydrogen the oxygen Auger signal decreased by $\approx 15 \%$ in a period of $\approx 2 \mathrm{~h}$ at $210^{\circ} \mathrm{C}$, whereas with hydrogen virtually all oxygen disappeared within $15 \mathrm{~min}$. No effect on $\Delta$ was observed due to hydrogen adsorbed on either the oxidised or the clean $\mathrm{Ni}$ crystal. The reaction rate, defined as $\left(\delta \Delta_{\max }\right)^{-1}$ $X \mathrm{~d}(\delta \Delta) / \mathrm{d} t$ on the straight line portion of the curves is proportional to the square

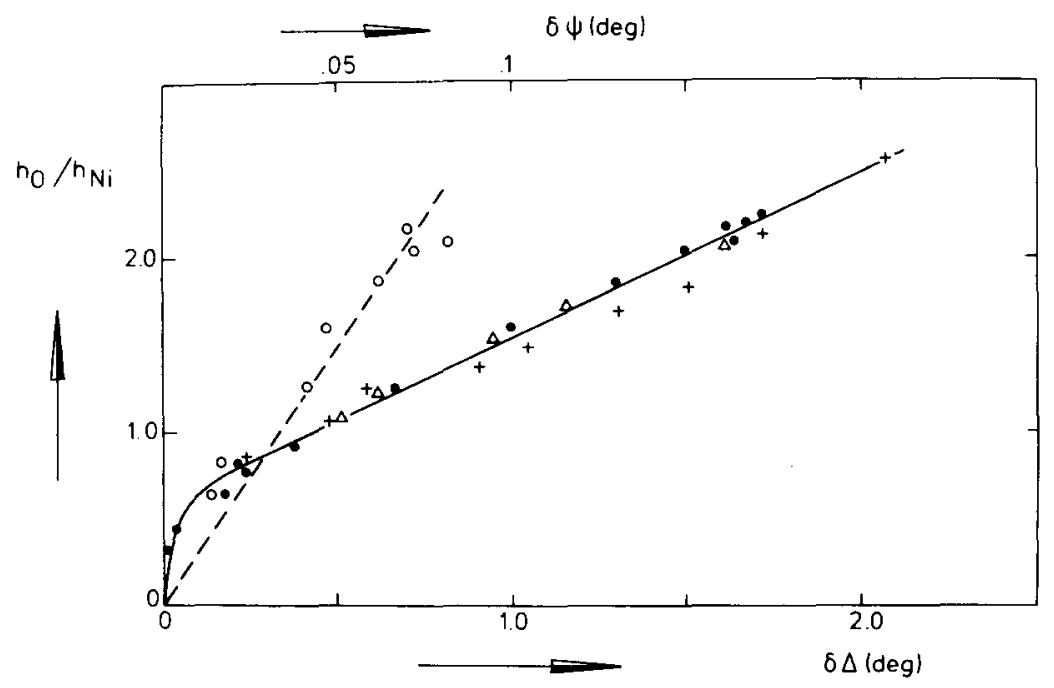

Fig. 2. Plot of the relative oxygen Auger signal $h_{\mathrm{O}}(515 \mathrm{eV}) / h_{\mathrm{Ni}}(848 \mathrm{eV})$ as a function of $\delta \Delta$ (lower scale) and $\delta \psi$ (upper scale, open circles). $\circ: \delta \psi$ at $25^{\circ} \mathrm{C} ; \bullet: \delta \Delta$ at $25^{\circ} \mathrm{C} ; \Delta: \delta \Delta$ at $50^{\circ} \mathrm{C}$; $+: \delta \Delta$ at $150^{\circ} \mathrm{C}$. 


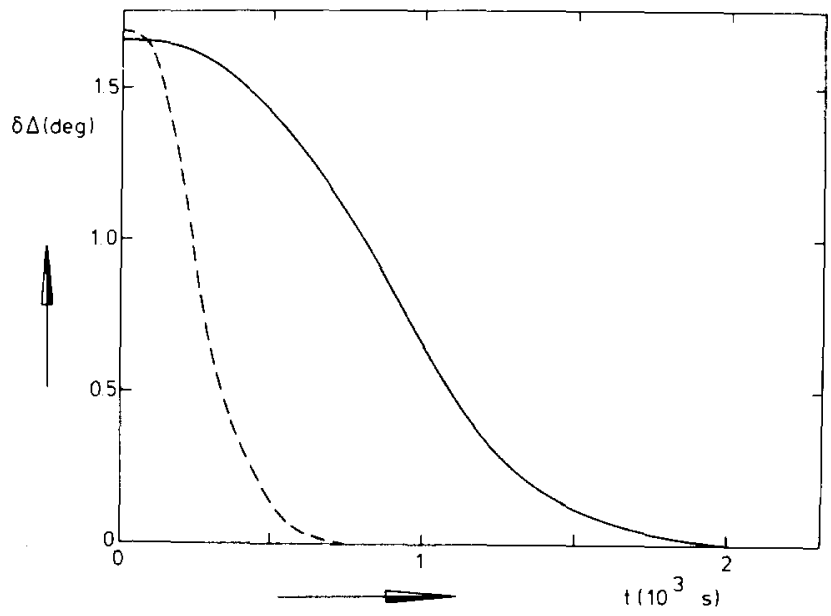

Fig. 3. Removal of oxygen from oxidised $\mathrm{Ni}(100)$ by $\mathrm{H}_{2}$ as determined by ellipsometry at $207^{\circ} \mathrm{C}$. Full curve: $p\left(\mathrm{H}_{2}\right)=2 \times 10^{-6}$ Torr; dashed curve: $p\left(\mathrm{H}_{2}\right)=18 \times 10^{-6}$ Torr.

root of the hydrogen pressure. In contrast to the oxidation reaction, the reduction of nickel oxide thus does not scale as exposure $(\mathrm{p} t)$. The reduction could be stopped at an arbitrary point on the curves of fig. 3 by evacuating the system. After readmission of hydrogen the reduction simply continued, i.e. there was no initial incubation period or horizontal part of the $\delta \Delta$ versus $t$ curve. An instantaneous decrease of oxygen coverage was also observed on reducing a partially oxidised $\mathrm{Ni}(100)$ surface. An incubation time for the reduction is hence exhibited by a fully oxidised surface only $(\delta \Delta \approx 1.7 \mathrm{deg})$.

LEED pictures taken at different degrees of surface oxidation showed the reported $p(2 \times 2)$ and $c(2 \times 2)$ structures in the chemisorption stage [1] followed by the formation of a complicated $\mathrm{NiO}$ overlayer [5] as illustrated in fig. 4. Upon admission of hydrogen this structure gradually reverted to a $c(2 \times 2)$ overlayer. After a slight reduction the $\mathrm{NiO}$ and $\mathrm{c}(2 \times 2)$ structures could be seen simultaneously. Finally, at $\delta \Delta \approx 0$, only the $c(2 \times 2)$ structure remained.

The $c(2 \times 2)$ structure could be transformed into the $\mathrm{p}(2 \times 2)$ pattern after prolonged hydrogen exposure $\left(\approx 10^{6} \mathrm{~L}\right)$, without altering the value of $\delta \Delta \approx 0$. This observation is consistent with the oxidation experiments, which show that the first half monolayer of oxygen can not be detected by ellipsometry at the chosen wavelength. Similarly, argon ion bombardment has been shown to change the $c(2 \times 2)$ structure into $\mathrm{p}(2 \times 2)[1]$. It is noteworthy, however, that even after exposures to hydrogen at temperatures around $200^{\circ} \mathrm{C}$ and pressures up to $10^{-4}$ Torr a small amount of oxygen always remained on the surface.

Finally we should mention that the reduction rate is enhanced by about an order of magnitude by switching on the Auger gun during hydrogen admission. On the 

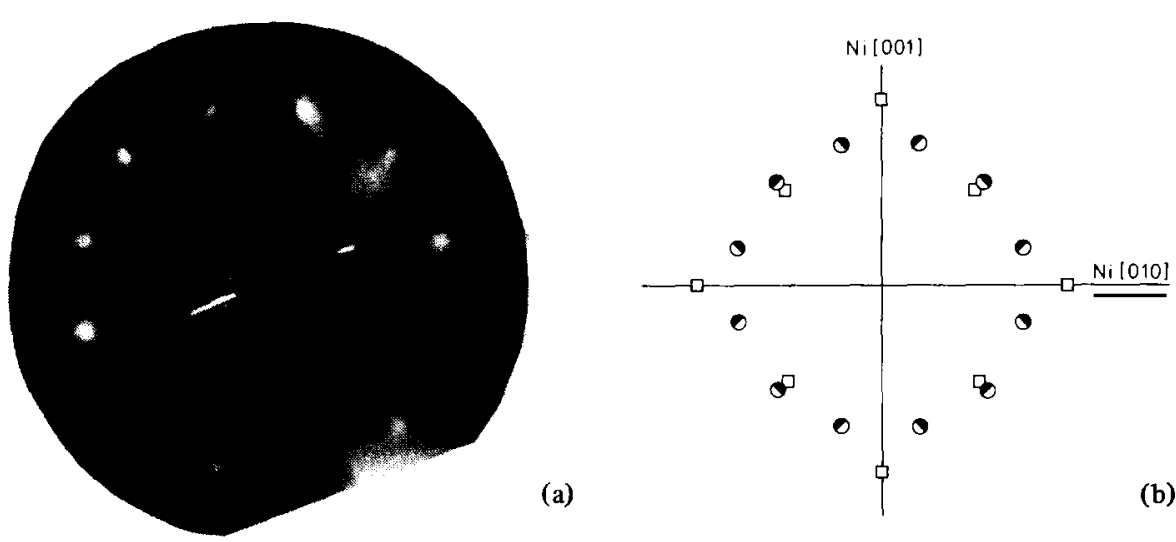

Fig. 4. (a) LEED pattern of fully oxidised $\mathrm{Ni}(100)$ at $98 \mathrm{eV}$ and $200^{\circ} \mathrm{C}$. (b) Assignment of diffraction pattern in (a), c.f. ref. [5]. $\mathrm{a}: \mathrm{NiO}(111) / / \mathrm{Ni}(100)$, $\mathrm{NiO}[\overline{1} 10] / / \mathrm{Ni}[011]$, $\bullet: \mathrm{NiO}(111) / /$ $\mathrm{Ni}(100)$, $\mathrm{NiO}[\overline{1} 10] / / \mathrm{Ni}[0 \overline{1} 1]$, a: $\mathrm{NiO}(100) / / \mathrm{Ni}(100), \mathrm{NiO}[001] / / \mathrm{Ni}[001]$ or $\mathrm{Ni}_{2} \mathrm{O}_{3}[001] / /$ $\mathrm{Ni}[001]$.

other hand, the reduction was much slower when the fully oxidised surface was left overnight or when the amount of oxygen was higher than corresponded to $\delta \Delta>2$ $\operatorname{deg}\left(>4 \times 10^{15} \mathrm{at} / \mathrm{cm}^{2}\right.$, vide infra). In the former case the LEED pattern also changed, showing simultaneously the $\mathrm{NiO}$ and $\mathrm{c}(2 \times 2)$ overlayer.

\section{Discussion}

The linearity between $\delta \Delta$ and the relative oxygen Auger signal in the incorporation stage (fig. 2) enables us to obtain the oxidation kinetics directly from the measured ellipsometric curves, provided we can take the relative oxygen Auger signal to be proportional to the surface coverage. This point has been discussed by Holloway and Hudson [1], who showed this to be approximately correct. The saturation coverage of oxygen at room temperature and pressures up to $10^{-4}$ Torr has been shown to be $3.32 \times 10^{15} \mathrm{at} / \mathrm{cm}^{2}$ [5], whence we obtain in the incorporation region

$N=2.0 \times 10^{15} \delta \Delta$,

where $N$ is the surface coverage in atoms per $\mathrm{cm}^{2}$.

Following earlier work [1,5] we describe the oxidation curves by an Avrami type of equation $[1,13]$

$N=N_{m}\left\{1-\exp \left(-\alpha p_{\mathrm{O}_{2}}^{n} t^{2}\right)\right\}$,

where $N_{m}$ is the saturation coverage, $\alpha$ a constant depending only on the temperature and $p_{\mathrm{O}_{2}}$ the gas pressure. The time dependence, $t^{2}$, has been assumed a priori 
Table 1

Values of $\alpha$ (eq. (2)) and induction times $t_{0}$ (expressed in $\mathrm{L}$ ) for the oxidation of $\mathrm{Ni}(100)$ at different temperatures

\begin{tabular}{|c|c|c|c|c|c|}
\hline \multirow[t]{2}{*}{$\begin{array}{l}T \\
\left({ }^{\circ} \mathrm{C}\right)\end{array}$} & \multicolumn{3}{|c|}{${ }^{\alpha}\left(10^{8}\right.$ Torr $\left.^{-2} \mathrm{~s}^{-2}\right)$} & \multicolumn{2}{|c|}{$\begin{array}{l}t_{0} \\
(\mathrm{~L})\end{array}$} \\
\hline & (a) & (b) & (c) & (b) & (c) \\
\hline 25 & 3.0 & - & 1.6 & - & 26 \\
\hline 40 & - & 1.0 & - & 30 & - \\
\hline 50 & - & - & 0.85 & - & 26 \\
\hline 100 & 1.2 & 0.3 & 0.61 & 40 & 56 \\
\hline
\end{tabular}

(a) ref. $[1] ;$ (b) ref. $[5] ;$ (c) this work.

as appropriate to two-dimensional growth [13]. A discussion of eq. (2) is given in the appendix. The exponent $n$ can be determined experimentally and is found to be equal to 2. Values of $\alpha$ are given in table 1 . Also included are the values of $t_{0}$ (expressed in $\mathrm{L}$ ), the time at which eq. (2) starts to be applicable. The values are in good agreement with those of other authors, which shows that our ellipsometric approach can be used with confidence to monitor the oxygen coverage in excess of half a monolayer. Fig. 5 shows a comparison between calculated and observed oxidation curves. The reduction of the surface oxide can be described by an equation

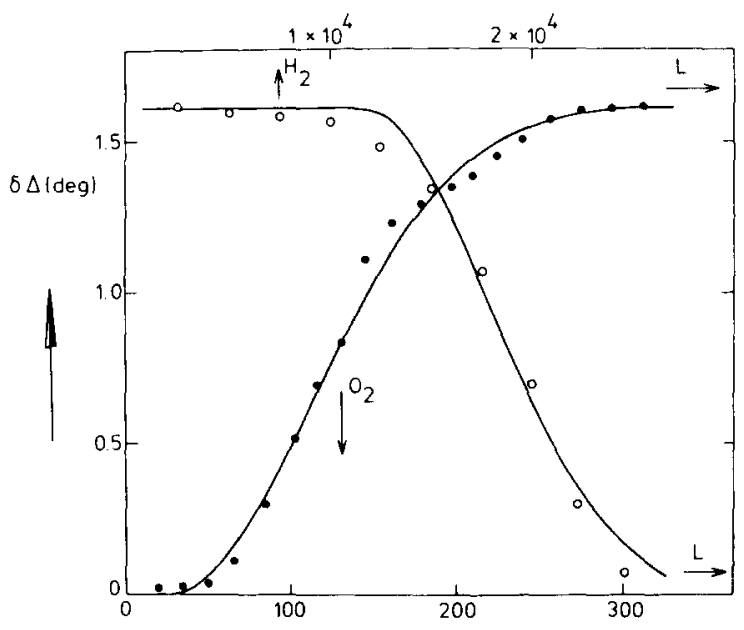

Fig. 5. Comparison of calculated (full lines) and observed kinetics of oxidation (lower scale) and reduction (upper scale) of $\mathrm{Ni}(100)$. $\bullet$ : oxidation at room temperature, $p\left(\mathrm{O}_{2}\right)=7.3 \times 10^{-8}$ Torr; $\circ$ : reduction of surface oxide by $\mathrm{H}_{2}$ at $187^{\circ} \mathrm{C}, p\left(\mathrm{H}_{2}\right)=3.2 \times 10^{-5}$ Torr. 
analogous to eq. (2):

$N=N_{m} \exp \left(-\beta p_{\mathrm{H}_{2}}^{m} t^{2}\right)$.

However, in contrast to the oxidation process, the reduction has $m=1$, as we have found by plotting the slope of the experimental curves at the inflection point

$\frac{1}{N_{m}}\left(\frac{\mathrm{d} N}{\mathrm{~d} t}\right)=-\left(\frac{2 \beta p_{\mathbf{H}_{2}}^{m}}{e}\right)^{1 / 2}$,

versus $p_{\mathrm{H}_{2}}^{1 / 2}$ at constant temperature. A plot of eq. (3) is also shown in fig. 5 .

Table 2 lists the values of $\beta$ at different temperatures and the value of the induction time $t_{0}$ (in L). As shown in the appendix a value of $m=1$ in eq. (3) results if hydrogen is dissociatively adsorbed on the $\mathrm{Ni} / \mathrm{NiO}$ surface and in equilibrium with the gas phase. Surface diffusion of $\mathrm{H}$ atoms or the actual $\mathrm{H}_{2} \mathrm{O}$ formation must then be rate limiting. In either case the existence of $\mathrm{H}$ atoms is required for the reduction to take place. This is corroborated by the experiments where the Auger gun was switched on during the reduction, which increased the reaction velocity considerably. The temperature dependence of $\beta$ (in Torr ${ }^{-1} \mathrm{~s}^{-2}$ ) may be expressed as

$\ln \beta=(25 \pm 2)-(11.7 \pm 1) \times 10^{3} / T$,

which yields an activation energy of $23 \mathrm{kcal} / \mathrm{mol}$. Due to the differences in reaction geometry, two-dimensional surface reduction versus reduction of a three-dimensional crystal, this value is difficult to compare with those obtained for the reduction of bulk $\mathrm{NiO}$ [9-11]. As shown in the appendix the activation energy is either given by $2 E_{\text {diff }}-E_{\mathrm{ad}}$ if surface diffusion is rate determining or $2 E_{\text {react }}-E_{\text {ad }}$ if the actual chemical reaction dictates the velocity of the process.

The problem now arises whether the active hydrogen atoms are actually adsorbed on exposed nickel atoms of the original nickel surface or on the nickel oxide overlayer. It has recently been shown by a quantum chemical calculation that $\mathrm{H}_{2}$ can only be dissociatively adsorbed on lattice defects on $\mathrm{NiO}$ [20]. The binding energy

Table 2

Values of $\beta$ (eq. (3)) and induction times (expressed in $L$ ) for the reduction of the surface nickel oxide by hydrogen

\begin{tabular}{llll}
$T$ & $\begin{array}{l}t_{0} \\
\left(10^{3} \mathrm{~L}\right)\end{array}$ & $\begin{array}{l}\beta \\
\left(\mathrm{Torr}^{-1} \mathrm{~s}^{-2}\right)\end{array}$ \\
\hline 160 & - & 0.047 \\
173 & 26 & 0.14 \\
190 & 12 & 0.22 \\
207 & 2.1 & 0.65 \\
212 & - & 1.33 \\
223 & 2.1 & 1.78 \\
232 & - & 1.96 \\
\hline
\end{tabular}


of a defect site is some $100 \mathrm{kcal} / \mathrm{mol}$ higher than on a perfect lattice site. Thus, since $E_{\text {diff }}$ can be estimated from ref. [20] to be about $6 \mathrm{kcal} / \mathrm{mol}$, the initial release of an $\mathrm{H}$ atom on a defect must be rate limiting. Since our observed activation energy is much less than $100 \mathrm{kcal} / \mathrm{mol}$ we see no way to reconcile this hypothesis with the experimental facts. We therefore conclude that the reduction reaction is not determined by hydrogen adsorbed on the nickel oxide. Alternatively one may assume hydrogen to be adsorbed dissociatively on the clean parts of the nickel surface once the reaction has started and some free nickel atoms have been exposed. This assumption yields, using $E_{\mathrm{ad}}=23 \mathrm{kcal} / \mathrm{mol}$ [21], an activation energy for diffusion of about $23 \mathrm{kcal} / \mathrm{mol}$ which is much larger than actually observed $\left(E_{\text {diff }}=7 \mathrm{kcal} / \mathrm{mol}\right.$ [22] $)$. The value of $E_{\mathrm{ad}}=23 \mathrm{kcal} / \mathrm{mol}$ pertains to adsorption of hydrogen on a pure nickel crystal. If the actual adsorption takes place on the $\mathrm{Ni}(100) \mathrm{c}(2 \times 2) \mathrm{O}$ overlayer (which remains after the reduction reaction) this value may be lower. However, even a value of $E_{\text {ad }}=0$ leads to $E_{\text {diff }}=11.5 \mathrm{kcal} / \mathrm{mol}$, which seems unreasonable. In this case the actual $\mathrm{H}_{2} \mathrm{O}$ formation must be rate limiting with an activation energy of $E_{\text {react }}$ between 11.5 and $23 \mathrm{kcal}$ but probably closer to the latter value.

We will now turn to the induction times, given in tables 1 and 2. From models for nucleation and growth one obtains the equation [23]

$\ln t_{0}=A+\frac{4 \pi v^{2} \sigma^{3} b}{3(k T)^{3} \ln ^{2}\left(p / p_{\infty}\right)}$,

where $A$ is a constant, $\sigma$ the surface tension of the new phase, $v$ the volume of a molecule, $p_{\infty}$ the equilibrium pressure for the reaction considered, $p$ the actual pressure in the experiment and $b$ a numerical factor $(0 \leqslant b \leqslant 1)$ referring to the differences between homogeneous and heterogeneous nucleation [23]. For the oxidation reaction we calculate, using bulk thermodynamic data for $\mathrm{NiO}, \sigma b^{1 / 3} \simeq 10^{3} \mathrm{erg} / \mathrm{cm}^{2}$, which leads to a surface tension of $\mathrm{NiO}$ of $10^{3}$ to $10^{4} \mathrm{erg} / \mathrm{cm}^{2}$, which is not unreasonable. For the reduction reaction eq. (6) is difficult to use since both the actual chemical reaction (reduction of $\mathrm{NiO}$ or $\mathrm{Ni}(\mathrm{OH})_{x}$ ) and the actual water pressure are unknown in our experiments. If therefore seems that not many conclusions can be drawn from the induction times. However, from Arrhenius plots of $\ln t_{0}$ apparent activation energies are obtained that are comparable to those obtained from a plot of the true rate constants $\alpha$ and $\beta(-2.4$ and $23 \mathrm{kcal} / \mathrm{mol}$, respectively).

The observed LEED pattern at saturation is composed mainly of two orientations of $\mathrm{NiO}(111)$ on the $\mathrm{Ni}(100)$ substrate: $\mathrm{NiO}[\overline{1} 10] / / \mathrm{Ni}[011]$ and $\mathrm{NiO}[\overline{1} 10] / / \mathrm{Ni}[0 \overline{1} 1]$, each giving rise to six diffraction spots as shown in fig. 4. At oxygen exposures greater than about $150 \mathrm{~L}$ very faint and diffuse diffraction spots appeared just inside those of the clean $\mathrm{Ni}(100)$ spots. These can be ascribed to $\mathrm{NiO}(100)$ or to a $\mathrm{Ni}_{2} \mathrm{O}_{3}$ surface oxide which has an identical lattice parameter $\left(\mathrm{Ni}_{x} \mathrm{O}_{y}[001] / / \mathrm{Ni}[001]\right)$. However, no firm evidence for the presence of $\mathrm{Ni}_{2} \mathrm{O}_{3}$ can be inferred from the present experiments, although photoemission measurements point to its existence [6]. Upon admission of hydrogen the whole LEED pattern shown in fig. 4 gradually disap- 
peared. This is in agreement with our assumed reaction mechanism (eq. (3)) which implies a continuous decrease of the surface area of nickel oxide islands.

\section{Conclusions}

(i) The reduction of $\mathrm{NiO}$ on $\mathrm{Ni}(100)$ with $\mathrm{H}_{2}$ requires the presence of (adsorbed) $\mathrm{H}$ atoms. These atoms are adsorbed on exposed clean nickel.

(ii) The reduction follows a nucleation and growth type mechanism with an overall activation energy of $23 \mathrm{kcal} / \mathrm{mol}$. The rate-limiting step is the formation of $\mathrm{H}_{2} \mathrm{O}$, also with an activation energy of $23 \mathrm{kcal} / \mathrm{mol}$.

\section{Appendix}

For a random distribution of germ nuclei the surface coverage at time $t, N(t)$, is related to the extended surface coverage $N_{\text {ext }}(t)$ by $[1,13]$ :

$N(t)=\left[1-\exp \left\{-N_{\text {ext }}(t)\right\}\right] N(\infty)$,

where $N_{\text {ext }}(t)$ is the hypothetical coverage that would be obtained if no overlap of growing islands is taken into account. For circular islands growing with constant radial velocity $\mathrm{d} r / \mathrm{d} t$ from $\chi_{0}$ nuclei, all present at $t=0, N_{\text {ext }}(t)$ is given by:

$N_{\text {ext }}(t)=\varkappa_{0} \pi(\mathrm{d} r / \mathrm{d} t)^{2} t^{2}$.

If direct impingement from the gas phase at the edges of the nuclei determines the radial growth velocity one has:

$\mathrm{d} r / \mathrm{d} t=p(2 \pi m k T)^{-1 / 2} \mathrm{~A}^{3 / 2}$,

where $A$ is the surface area of an atom. If surface diffusion is rate determining one derives easily

$\mathrm{d} r / \mathrm{d} t=D_{\mathrm{s}}(\mathrm{A} / l) c_{\mathrm{s}}$,

where $D_{\mathrm{s}}$ is the surface diffusion coefficient, $l$ the distance over which the concentration on the surface changes from its unperturbed value $c_{s}$ to zero on the perimeter of the island $\left(l \approx \mathrm{A}^{1 / 2}\right)$. If the actual island growth is rate determining with rate constant $k$ one has:

$\mathrm{d} r / \mathrm{d} t=k \mathrm{~A}^{1 / 2} c_{\mathrm{s}}$.

The previous expressions differ from those given in ref. [1] due to the somewhat different assumptions we have made about the diffusion process. The important point, however, is that eq. (A.3) predicts a proportionality between the growth rate and pressure, whereas the other two limiting cases have a proportionality between growth rate and surface coverage. If, for example, dissociative adsorption prevails 
and equilibrium between gas phase and adsorbed phase is assumed, we have

$c_{\mathrm{S}}=\left(k_{\mathrm{ad}} p\right)^{1 / 2}$,

where $k_{\mathrm{ad}}=k_{\mathrm{ad}}^{0} \exp \left(E_{\mathrm{ad}} / R T\right)$, with $E_{\mathrm{ad}}$ the heat of adsorption. Hence

$\mathrm{d} r / \mathrm{d} t=D_{\mathrm{s}}(\mathrm{A} / l)\left(k_{\mathrm{ad}} p\right)^{1 / 2}$,

from eq. (A.4), or, from eq. (A.5)

$\mathrm{d} r / \mathrm{d} t=k \mathrm{~A}^{1 / 2}\left(k_{\mathrm{ad}} p\right)^{1 / 2}$.

For the reduction reaction the growth of nickel islands not covered by oxygen is described by eq. (A.1) and thus the oxygen coverage by

$N(t)=N(0) \exp \left\{-N_{\text {ext }}(t)\right\}$.

Substituting either eq. (A.7) or (A.8) into eqs. (A.2) and (A.9) gives

$N(t)=N(0) \exp \left\{-\varkappa_{0} \pi D_{\mathrm{s}}^{2}(\mathrm{~A} / l)^{2} k_{\mathrm{ad}} p t^{2}\right\}$

or

$N(t)=N(0) \exp \left\{-\pi_{0} \pi k^{2} \mathrm{~A} k_{\mathrm{ad}} p t^{2}\right\}$.

Arrhenius equations for the diffusion, reaction and adsorption rate constants lead to experimental activation energies for $\beta$ (see eqs. (3) and (4)) given by

$E_{\text {exp }}=-E_{\text {ad }}+2 E_{\text {diff }}$ (diffusion rate-limiting),

or

$E_{\mathrm{exp}}=-E_{\mathrm{ad}}+2 E_{\text {react }}$ (reaction rate-limiting).

\section{References}

[1] P.H. Holloway and J.B. Hudson, Surface Sci. 43 (1974) 123.

[2] P.H. Holloway and J.B. Hudson, Surface Sci. 43 (1974) 141.

[3] J.E. Demuth and T.N. Rhodin, Surface Sci. 45 (1974) 249.

[4] N.G. Krishnan, W.N. Delgass and W.D. Robertson, Surface Sci. 57 (1976) 1.

[S] D.F. Mitchell, P.B. Sewell and M. Cohen, Surface Sci. 61 (1976) 355.

[6] P.R. Norton, R.L. Tapping and J.W. Goodale, Surface Sci. 65 (1977) 13.

[7] T. Fleisch, N. Winograd and W.N. Delgass, Surface Sci. 78 (1978) 141.

[8] K. Akimoto, Y. Sakisaka, M. Nishijama and M. Onchi, Surface Sci. 82 (1978) 349.

[9] K. Hauffe and A. Rahmel, Z. Phys. Chem. Frankfurt 1 (1954) 104.

[10] B. Delmon, Bull. Soc. Chem. Fr. (1961) 590.

[11] W. Pluskell and B.V. Sarma, Arch. Eisenhüttenwes. 45 (1974) 23.

[12] F. Chiesa and M. Rigaud, Can. J. Chem. Eng. 49 (1971) 617.

[13] M. Avrami, J. Chem. Phys. 7 (1939) 1103; 8 (1940) 212;9 (1941) 177.

[14] Y. lida and K. Shimada, Bull. Chem. Soc. Japan 33 (1960) 790.

[15] H. Albers, Thesis, University of Utrecht (1978). 
[16] F.H.P.M. Habraken, Thesis, University of Utrecht (1980).

[17] F.H.P.M. Habraken, O.L.J. Gijzeman and G.A. Bootsma, Surface Sci., to be published.

[18] F.C. Schouten, E.W. Kaleveld and G.A. Bootsma, Surface Sci. 63 (1977) 460.

[19] F.C. Schouten, O.L.J. Gijzeman and G.A. Bootsma, Surface Sci. 87 (1979) 1.

[20] G.T. Surrat and A.B. Kunz, Phys. Rev. B19 (1979) 2352.

[21] J. Lapujoulade and K.S. Neil, Surface Sci. 35 (1973) 288.

[22] R. Gomer, Discuss. Faraday Soc. 28 (1959) 23.

[23] L.I. Klochina, T.N. Naumova, S.N. Bulatov and I.A. Leferov, Russ. J. Phys. Chem. 50 (1976) 1729. 